ARTICLE

\title{
Hydrogen radical-shuttle (HRS)-enabled photoredox synthesis of indanones via decarboxylative annulation
}

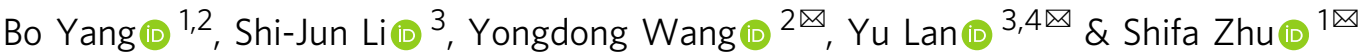

Hydrogen atom transfer (HAT) process is a powerful and effective strategy for activating $\mathrm{C}-\mathrm{H}$ bonds followed by further functionalization. Intramolecular $1, n$ ( $n=5$ or 6 )-HATs are common and frequently encountered in organic synthesis. However, intramolecular $1, n$ ( $n=2$ or 3)-HAT is very challenging due to slow kinetics. Compared to proton-shuttle process, which is well established for organic synthesis, hydrogen radical-shuttle (HRS) is unexplored. In this work, a HRS-enabled decarboxylative annulation of carbonyl compounds via photoredox catalysis for the synthesis of indanones is developed. This protocol features broad substrate scope, excellent functional group tolerance, internal hydrogen radical transfer, atom- and step-economy. Critical to the success of this process is the introduction of water, acting as both HRS and hydrogen source, which was demonstrated by mechanistic experiments and density functional theory (DFT) calculations. Importantly, this mechanistically distinctive HAT provides a complement to that of typical proton-shuttle-promoted, representing a breakthrough in hydrogen radical transfer, especially in the inherently challenging 1,2- or 1,3HAT.

\footnotetext{
${ }^{1}$ Key Laboratory of Functional Molecular Engineering of Guangdong Province, School of Chemistry and Chemical Engineering, South China University of Technology, Guangzhou, China. ${ }^{2}$ Singfar Laboratories, Guangzhou, China. ${ }^{3}$ Green Catalysis Center, and College of Chemistry, Zhengzhou University, Zhengzhou, Henan, China. ${ }^{4}$ School of Chemistry and Chemical Engineering, and Chongqing Key Laboratory of Theoretical and Computational Chemistry,

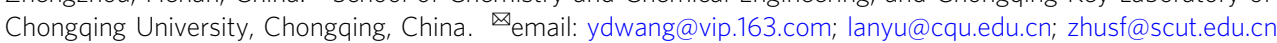


A s a powerful and effective strategy, hydrogen atom transfer (HAT) catalysis has been demonstrated as an ideal platform for $\mathrm{C}-\mathrm{H}$ bonds functionalizations, majorly involving proton shift and hydrogen radical transfer ${ }^{1-12}$. When it comes to proton transfer, proton-shuttle (PS) catalysis has been well developed for the past decades, providing a highly efficient strategy for $\mathrm{C}-\mathrm{H}$ functionalization, especially in transition-metalcatalyzed $\mathrm{C}-\mathrm{H}$ activation ${ }^{13-15}$ and insertion of carbenes into heteroatom-hydrogen bonds ${ }^{16-24}$ (Fig. 1a). It has been recognized that PS catalysts, such as water ${ }^{16}$, acid ${ }^{13}, 17-23$ and alcohol ${ }^{24}$, could lower the reaction barrier by forming cyclic molecular complexes that involve lower ring strain and facilitate intra- or intermolecular HAT. In the latter process (hydrogen radical transfer), a reactive radical species, traditionally, was needed to abstract hydrogen from $\mathrm{C}-\mathrm{H}$ bond to generate the corresponding carbon-centered radical intermediate ${ }^{6-8}, 11,25-28$, triggering the following functionalization process. Based on the great achievements in PS catalysis, we wondered whether a similar hydrogen radical-shuttle (HRS) strategy could be used to complete the HAT process (Fig. 1b). Notably, the core difference between HRS-promoted HAT and that of polarity-reversalcatalyzed $^{29,30}$ is that hydrogen radical transfer occurs from a neutral position to another non-radical site. To the best of our knowledge, no successful examples utilizing this strategy have been reported. Importantly, this would be another complementary process to that of PS catalysis. With this HRS strategy in mind, we engaged to develop practical approaches for important scaffolds synthesis via a radical pathway.
Considering the prevalence of indanones and their derivatives in pharmaceuticals and biologically active natural products ${ }^{31-35}$, a lot of efforts have been devoted to developing effective strategies for indanones synthesis ${ }^{36-40}$. Traditionally, indanones were prepared from the corresponding indenols or indenones. Among a variety of approaches, transition-metal catalyzed annulation of ortho-halogenated carbonyl compounds and alkynes is one of the highly efficient and general strategies to construct indenone scaffolds $^{41-45}$. For example, Yamamoto ${ }^{46}$ and Cheng ${ }^{47,48}$ reported the cyclization of ortho-halogenated carbonyl compounds and alkynes to construct indenols, respectively. Kong ${ }^{49}$ reported the indanones synthesis based on hydrogen autotransfer strategy through nickel catalysis. Notwithstanding great achievements that have been made, these methods typically suffered from the prefunctionalization of the corresponding starting materials. Direct $\mathrm{C}-\mathrm{H}$ bond functionalization to access indenones through $\mathrm{Rh}$-catalyzed procedures has also been developed ${ }^{50-52}$. However, among these traditional strategies, a stepwise process has to be adopted because additional oxidation and/or reduction processes are often required when converting the indenols or indenones to indanones (Fig. 1c). Therefore, developing a direct $\mathrm{C}-\mathrm{H}$ annulation of carbonyl compounds with alkynes for indanones synthesis in one step is highly appealing and desirable.

Recently, aryl $\mathrm{Csp} p^{2}-\mathrm{H}$ functionalization involving a radical process has emerged as an ideal and powerful strategy to construct C-C bonds, along with diminished cost and waste ${ }^{53,54}$. These methods rely on certain carbon radicals trapped by arenes and followed by the aromatization process, which might provide a Proton-shuttle catalysis (PS): well-known

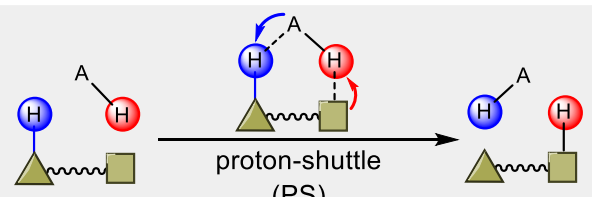

(PS) b Hydrogen radical-shuttle catalysis (HRS): unknown

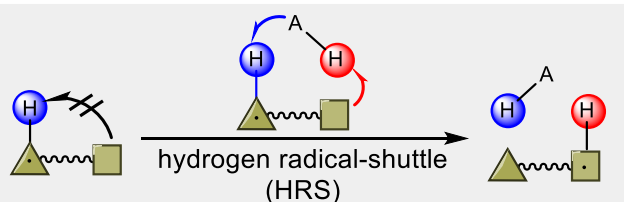

c Previous strategies for the synthesis of indanones (stepwise)
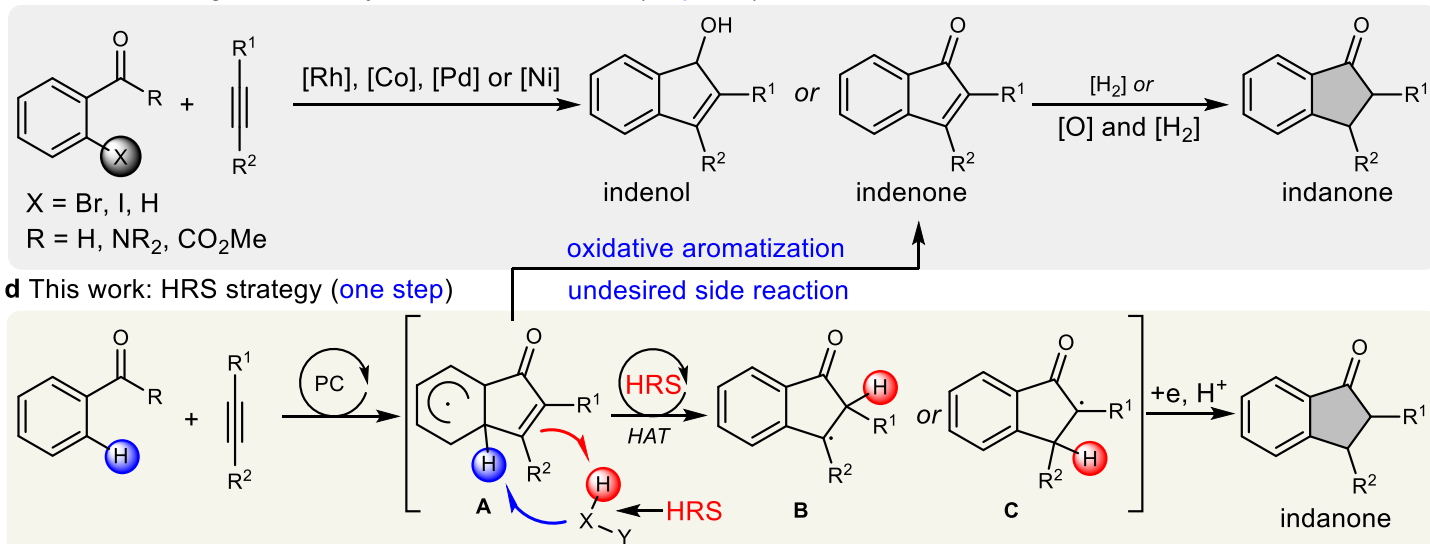

Challenges:

- Direct addition of radical to alkyne is kinetically slow

- The generated vinyl radical is highly reactive

- Intermediate $A$ is prone to form indenone through oxidative aromatization

- Direct 1,2- or 1,3-HAT is extremely challenging
Features:

- Merger of photocatalysis with HAT

- Forbidden internal HAT process enabled by HRS

- No need for prefunctionalization of starting material

- Step-economy (one step)

- Synthesis of natural products and bioactive molecules

Fig. 1 Proton/hydrogen radical-shuttle catalysis and indanone scaffold synthetic strategy. a Proton-shuttle catalysis model. b Hydrogen radical-shuttle catalysis model. c Traditional synthetic strategies for indanones by annulation reaction of alkynes. d HRS-enabled strategy for indanone synthesis (this work). PC photocatalyst. 
an alternative protocol for the direct annulation of carbonyl compounds to construct indanones. In addition, acyl radicals produced efficiently from $a$-oxocarboxylic acid, aldehyde, acyl halide, and so on via radical pathway ${ }^{55}$, have been well researched with alkenes. Inspired by these developments, we anticipated that if we could utilize the electron-deficient vinyl radicals, generated from acyl radical addition to alkynes, to achieve direct construction of indanones through the dearomatic radical intermediate A. However, the typical oxidative aromatization strategy from intermediate $\mathbf{A}$ to the desired indanone product is often problematic due to (i) the electron-deficient indenone is readily prone to $[2+2]$ cycloaddition under photoredox conditions $s^{56}$ and (ii) the Giese-type reaction of acyl radical with indenone would be the main side reaction ${ }^{57}$. Moreover, the following external reduction steps were also required from indenone to indanone. To overcome these obstacles, we questioned if it is possible to merge HAT with single electron transfer (SET), resulting in the generation of intermediate $\mathbf{B}$ or $\mathbf{C}$. We recognized that such a merger might realize rearomatization of intermediate A and avoid the generation of indenone, providing an aromatization model and an ideal strategy for the direct construction of indanones without additional prefunctionalization of substrates and external steps. However, the direct addition of radicals to unactivated alkynes is kinetically slow ${ }^{58,59}$ and the generation of corresponding high-energy vinyl radical intermediate is highly reactive, which can participate in various undesirable open-shell pathways. In addition, intermediate $\mathbf{A}$ is prone to form indenone through oxidation/elimination steps. More importantly, as the critical problem in our design, the HAT $(1,2-$ or $1,3-\mathrm{HAT})$ strategy forming $\mathbf{B}$ or $\mathbf{C}$ is challenging $11,60,61$ due to the high activation energy, which could be attributed to the increased $\mathrm{C}-\mathrm{H}-\mathrm{C} /$ heteroatom strain. According to the analysis above, an HRS-enabled HAT strategy, we speculated, might be an ideal protocol to circumvent this problem (Fig. 1d). A suitable HRS catalyst was required to modulate the reactivity of intermediate $\mathbf{A}$, thereby providing an opportunity for rearomatization and hydrogen radical transfer of $\mathbf{A}$ simultaneously, furnishing the effective synthesis of indanones.

In this work, we report an HRS-enabled decarboxylative annulation of carbonyl compounds for the synthesis of indanones via photocatalysis with excellent functional group tolerance, broad substrate scope as well as an atom- and step-economy. The key to the success of this protocol is the application of water molecules, functioning as both solvent and HRS and promoting the hydrogen radical transfer in formal 1,3-HAT process, which was demonstrated by mechanistic experiments and DFT calculations.

\section{Results and discussion}

Reaction development. From a design perspective, with benzoylformic acid $\mathbf{1}$ as acyl radical precursor, we envisioned that this HRS-promoted HAT/SET strategy could be outlined as Fig. 2a. Irradiation of photocatalyst PC (I) with visible light generates the long-lived excited state II, which is a strong oxidant, capable of oxidizing 2 to form a nucleophilic acyl radical $3^{57}$ and a reduced state III. Meanwhile, the alkyne 4 reacts readily with acyl radical $\mathbf{3}$ to form the vinyl radical $\mathbf{5}$. The open-shell radical $\mathbf{5}$ is expected to rapidly engage in addition to the aryl ring, generating the dearomatic radical 6 . At this stage, we hoped that this radical species 6 would undergo the critical hydrogen radical transfer step to generate key intermediate 7 or $7^{\prime}$ assisted by HRS. Single-electron reduction of radical 7 or $7^{\prime}$ by III to afford carbon anion $\mathbf{8}$ or $\mathbf{8}^{\prime}$, followed by protonation to afford the indanone $\mathbf{9}$.

Our initial efforts sought to evaluate different potential HRSs which are effective for the direct assemble of indanones with potassium 2-oxo-2-phenylacetate 10 and phenylacetylene $\mathbf{4}$ as model substrates, along with $\operatorname{Ir}\left[\mathrm{dF}\left(\mathrm{CF}_{3}\right) \mathrm{ppy}\right]_{2}$ (phen) $\mathrm{PF}_{6}$ as the photocatalyst under $\mathrm{N}_{2}$ with illumination by blue LEDs. After a series of explorations on several potential HRS catalysts $\left(\mathrm{H}_{2} \mathrm{O}\right.$, $\mathrm{MeOH}, \mathrm{EtOH}$, and acetic acid), to our delight, $22 \%$ yield of indanone was isolated with water as an additive (Fig. 2b). A trace amount of desired indanone 9 was detected without water.

Further screening of the reaction conditions using benzoylformic acid $\mathbf{1}$ and phenylacetylene $\mathbf{4}$ as model substrates found that the indanone 9 could be isolated in $86 \%$ yield using $\operatorname{Ir}\left[\mathrm{dF}\left(\mathrm{CF}_{3}\right)\right.$ ppy $]_{2}$ (phen) $\mathrm{PF}_{6}$ as the photocatalyst and water as HRS under $\mathrm{N}_{2}$ with illumination by blue LEDs at $100^{\circ} \mathrm{C}$ (Fig. 2c). Control experiments revealed that the photocatalyst, visible light, and water were all essential components for achieving the high efficiency of this reaction (see Supplementary information for details).

Substrate scope investigation. With the optimized conditions in hand, we next evaluated the variations of 2-oxo-2-arylacetic acids and alkynes that are applicable to the developed reaction (Fig. 3). With respect to the 2-oxo-2-arylacetic acid partner, we observed moderate to excellent yields of the desired products (11-31) with a wide range of substrates bearing different substituents. The methyl groups at the ortho-, meta-, and para-positions on the phenyl ring of 2-oxo-2-arylacetic acid could be tolerated (11-13). With respect to the meta-substituted 2-oxo-2-phenylacetic acid, regioisomers 12 and $\mathbf{1 2}{ }^{\prime}$ were obtained with $0.75 / 1 \mathrm{rr}$. A range of 2-oxo-2-phenylacetic acids bearing both the electron-donating and electron-withdrawing substituents on the phenyl ring, no matter for $1^{\circ}-, 2^{\circ}-, 3^{\circ}$-alkyl substituents or linear, cyclic substituents, were amenable substrates (14-24). The strong electronwithdrawing substituents decrease the conversion and yields. This observation may be ascribed to the reduced reductive quenching ability toward photoexcited [Ir $]^{*}$. The $s p^{2}$-hybridized phenylsubstituted 2-oxo-2-phenylacetic acid underwent smoothly to give a $65 \%$ yield of indanones (25). Notably, 2-oxo-2-phenylacetic acid with additional functionalities was also compatible with this protocol. For example, various functional groups, such as ether, halides, trifluoromethyl, easily-oxidized thioether, ester, and amide remain intact to furnish the corresponding products (26-29). In addition to substituted 2-oxo-2-phenylacetic acidtype substrate, 2-(naphthalen-2-yl)-2-oxoacetic acid could also be successfully converted into the desired product $\mathbf{3 0}$ in reasonable yield. Interestingly, $\mathbf{3 1}$ and $\mathbf{3 1} \mathbf{1}^{\prime}$ could be obtained in $83 \%$ yield with region-selectivity $(r r 1 / 2)$ from the corresponding substrates. Having established that this transformation tolerates various 2oxo-2-arylacetic acid substrates, we then turned our attention towards evaluating the scope of the alkyne components. For the simple aromatic alkynes with alkyl or phenyl substituents, the corresponding products (32-37) were isolated in $43-84 \%$ yields. Evaluation of a series of alkynes that contained various functional groups, such as fluoro, chloro, bromo, nitrile, aldehyde, ketone, ester, acid, phenol, free amine and alcohol, provided indanones $38-48$ in $43-85 \%$ yield, potentially allowing for the subsequent orthogonal functionalization. Notably, alkynes with synthetic handles, such as halides (38-40) and boronic ester 49, were readily incorporated into the accessible indanone scaffolds, which highlights their potential applications for the incorporation of these scaffolds into more complex targets. Additionally, the developed protocol was also tolerant of the alkyne containing easily oxidized thioether, as demonstrated by $\mathbf{5 0}$, which was isolated in 63\% yield. Considering that heteroaryl-substituted compounds are highly desirable building blocks in drug discovery, we also evaluated a range of heteroaryl-substituted alkynes that would provide access to heteroaryl-substituted 


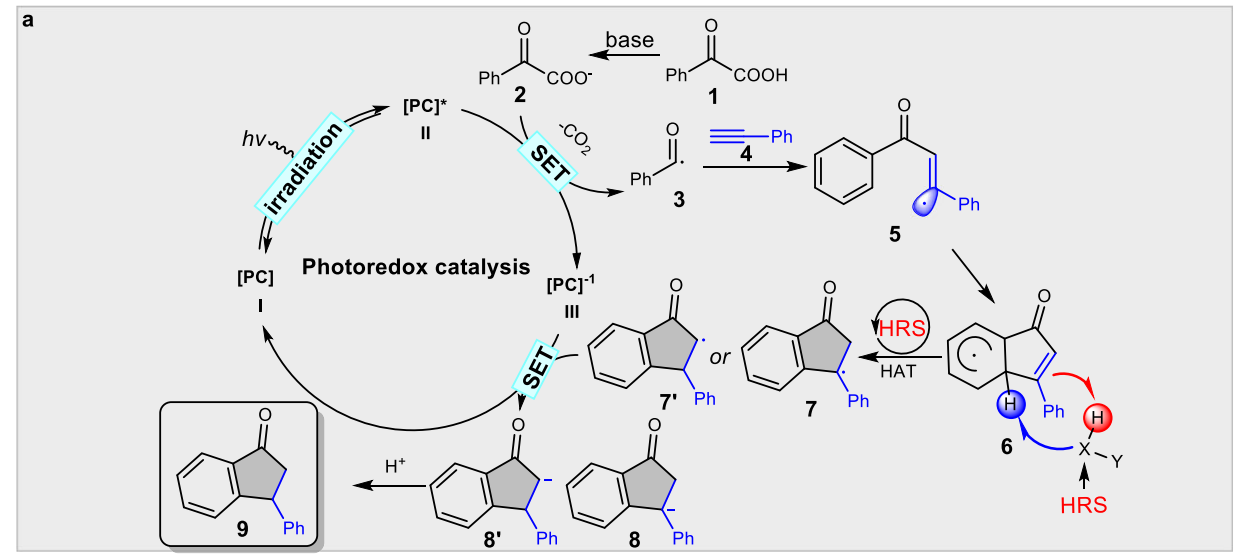

b

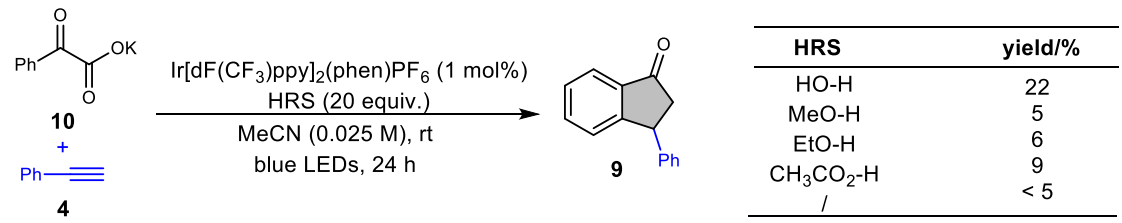

c

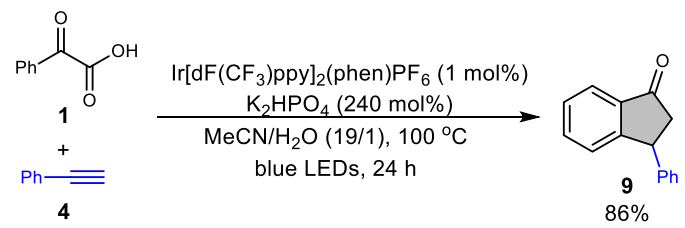

\begin{tabular}{cc}
\hline Temperature & yield/\% \\
\hline 60 & 58 \\
70 & 66 \\
80 & 70 \\
90 & 74 \\
100 & 86 \\
110 & 84 \\
\hline
\end{tabular}

Fig. 2 Reaction development. a Proposed mechanism for synthesis of indanone. b Evaluation of possible HRS catalyst. c Investigation of temperature's effect on the reaction. PC photocatalyst, HRS hydrogen radical-shuttle.

indanones. Although these scaffolds traditionally required multistep syntheses, the developed protocol allows for the construction of heteroaryl-substituted indanones in a single step from readily available precursors. For example, a wide range of fiveand six-membered heteroaryl alkynes, such as benzofuran, thiophene, indole, and pyridine-derived substrates were functionalized with high efficiency (51-55). When 2-naphthyl alkyne was subjected to the standard conditions, a reaction occurred to afford the desired product $\mathbf{5 6}$ in $69 \%$ yield. Of particular note is that when 1,4-diethynylbenzene was subjected to the standard conditions, mono-cyclization product 57 could be obtained in $20 \%$ yield, accompanied with an equal amount of dicyclization product 58. Dicyclization product $\mathbf{5 8}$ could be selectively produced in $61 \%$ yield with $1.1 / 1 d r$ when an excess amount of acid partner was used. Moreover, tricyclization compound $\mathbf{5 9}$ could be obtained in one step under the same reaction conditions by using 1,3,5-triethynylbenzene as an alkyne component. To further explore the scope of this reaction, other kinds of alkynes were also tested. The silicon-substituted alkynes and methyl propiolate were also suitable substrates to provide the titled products (60-61). Besides the terminal alkynes, the internal alkynes, including aromatic alkynes and alkyl alkynes, could also be successfully transformed into 2,3-disubstituted indanones with diastereometric ratios ranging from $7.2 / 1$ to $20 / 1$ (62-68). We also evaluated 2-oxo-2-phenylacetic acids and aromatic alkynes both with electron-donating substituents under standard conditions, providing the corresponding products in $67-80 \%$ yield $(69-71)$. Importantly, the reaction could be reproduced on a $6 \mathrm{mmol}$ scale to provide gram quantities of $\mathbf{7 1}$ in an increased concentration. There was almost no change in the chemical yield, suggesting that large-scale chemical production might be possible. It is noteworthy that cyclic internal alkyne, cyclooctyne, could also be transformed to the corresponding indanone $\mathbf{7 2}$ in $30 \%$ yield with $3.7 / 1 d r$.

To explore its utility for late-stage functionalization of complex molecules, several natural products or bioactive moleculesderived alkynes were tested for this developed reaction system. As shown in Fig. 4, the estrone-derived alkyne could be efficiently transformed into the indanone $\mathbf{7 3}$ in a $65 \%$ yield. In addition, aryl alkyne with an ester-linked androstrone also participated in this transformation smoothly, furnishing $\mathbf{7 4}$ in $75 \%$ yield. Similarly, the corresponding alkyne derived from menthol and adamantanol were both suitable alkyne partners for this protocol, affording the desired products $\mathbf{7 5}$ and $\mathbf{7 6}$ in $85 \%$ and $77 \%$ yield, respectively. These results show great potential for the structural modification of an array of complex biological molecules in medicinal chemistry.

To further showcase the synthetic utility of this developed strategy, we next made efforts on the synthesis of indanonecontaining natural products, biologically and pharmaceutically molecules. For example, the 3-substituted indanone-1-one 78, prepared using this method in $63 \%$ yield, was the key intermediate in the synthesis of indatraline $\mathbf{7 9 ^ { 6 2 }}$, an approved and antidepressant drug (Fig. 5a). Moreover, synthesis of PPAR $\gamma$ agonist $\mathbf{8 4}^{63}$ could be achieved via oxidated dehydrogenation of corresponding indanone $\mathbf{8 3}$, which was prepared in three steps from a-oxocarboxylic acid $\mathbf{8 0}$ and 4 using our developed protocol, followed by dehalogenation and $\alpha$-esterification (Fig. 5b). Importantly, pauciflorol F $\mathbf{8 7}^{64}$ and isopauciflorol F $90^{65}$, both are natural products and bioactive molecules, could also be selectively assembled by using different alkynes and aryl halides (Fig. 5c and d).

With the indanone scaffolds in hand, further chemical transformations were also performed to demonstrate the potential 


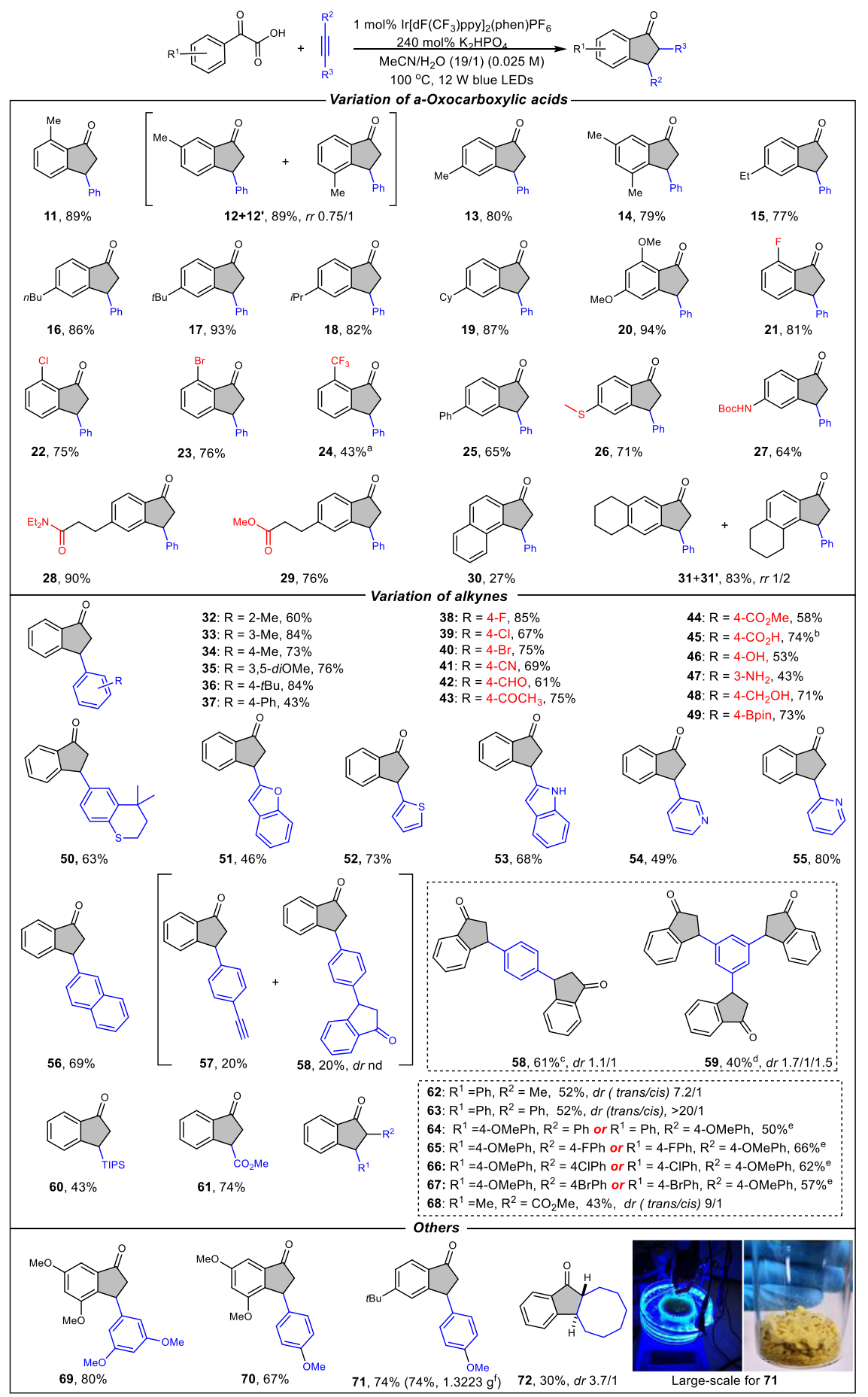

Fig. 3 Exploration of substrate scope. Reactions were performed with acid $(1.0 \mathrm{mmol})$, alkyne $(0.5 \mathrm{mmol}), \operatorname{Ir}\left[\mathrm{dF}\left(\mathrm{CF}_{3}\right) \mathrm{ppy}\right]_{2}(\mathrm{phen}) \mathrm{PF}_{6}(1 \mathrm{~mol} \%), \mathrm{K}_{2} \mathrm{HPO}_{4}$ (2.4 equiv.) $\mathrm{MeCN}\left(19 \mathrm{~mL}\right.$ ) and $\mathrm{H}_{2} \mathrm{O}(1 \mathrm{~mL}) .24 \mathrm{~h}, 100^{\circ} \mathrm{C}, 12 \mathrm{~W}$ blue LEDs. Isolated yields. Regioselectivity ratio (rr) determined by ${ }^{1} \mathrm{H} \mathrm{NMR.}{ }^{a} 48 \mathrm{~h}$. ${ }^{b} \mathrm{~K}_{2} \mathrm{HPO}_{4}$ (3.4 equiv.) was used. calkyne $(0.25 \mathrm{mmol})$ was used. dalkyne $(0.167 \mathrm{mmol})$ was used. ea mixture of regio- and diastereo-isomers, which is difficult to isolate. facid $(12.0 \mathrm{mmol})$, alkyne $(6.0 \mathrm{mmol}), \mathrm{MeCN}(120 \mathrm{~mL}), \mathrm{H}_{2} \mathrm{O}(6 \mathrm{~mL}), 48 \mathrm{~h}$.

applications of these molecules (Fig. 6). Taking indanone 9 as an example, the terminal alkene 91, 3-phenyl-1H-inden-1-one 92, lactone 93, and 1-phenyl-2,3-dihydro- $1 H$-indene 94 could be obtained via the Wittig reaction, oxidation by 2,3-dichloro-5,6dicyano-1,4-benzoquinone (DDQ), Baeyer-Villiger oxidation, and reduction by $\mathrm{Zn} / \mathrm{HOAc}$ system, respectively. The methylene group of 9 reacted with an aldehyde to give $(E)$-2-benzylidene-3phenyl-2,3-dihydro-1H-inden-1-one 95 efficiently. Particularly, benzocycloheptenone 96 can be prepared straightforwardly via the two-carbon ring expansion strategy with inexpensive ethylene developed by Dong's group ${ }^{66}$.

Given that the robust efficiency observed, we next turned our attention to a deeper exploration of the chemo- and regioselectivity of this water-mediated reaction by including different type $\mathrm{C}-\mathrm{H}$ bonds within various molecular probes (Fig. 7). An initial competition between multiple aryl $\mathrm{C}-\mathrm{H}$ bonds within a single intermediate illustrates the absolute propensity for generation of indanone 98 (98 vs. 99). This observation indicates 

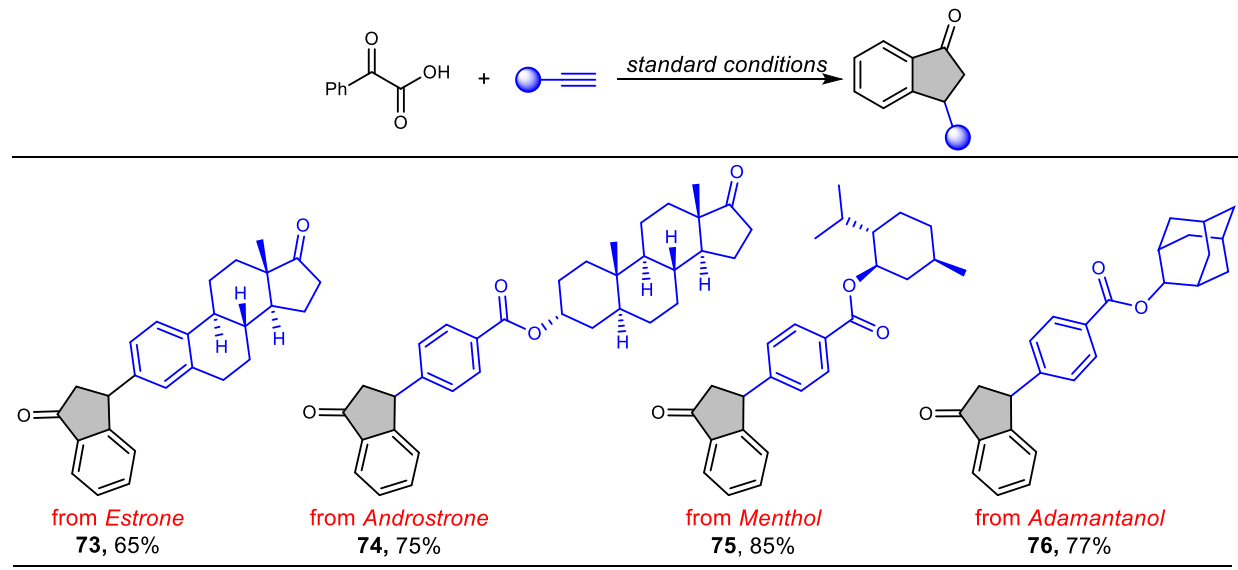

Fig. 4 Late-stage functionalization of complex molecules. Reactions conditions: acid $(1.0 \mathrm{mmol})$, alkyne $(0.5 \mathrm{mmol}), \operatorname{lr}\left[\mathrm{dF}\left(\mathrm{CF}_{3}\right) \mathrm{ppy}\right]_{2}(\mathrm{phen}) \mathrm{PF} \mathrm{F}_{6}(1 \mathrm{~mol} \%)$, $\mathrm{K}_{2} \mathrm{HPO}_{4}$ (2.4 equiv.) $\mathrm{MeCN}(19 \mathrm{~mL})$ and $\mathrm{H}_{2} \mathrm{O}(1 \mathrm{~mL}) .24 \mathrm{~h}, 100^{\circ} \mathrm{C}, 12 \mathrm{~W}$ blue LEDs. Isolated yields. The diastereometric ratio could not be determined by ${ }^{1} \mathrm{H}$ NMR analysis.

a<smiles>O=C(O)C(=O)c1ccccc1</smiles>

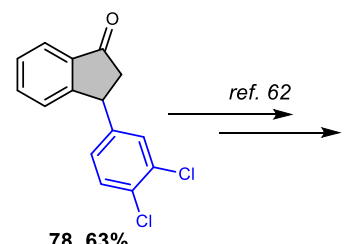

$78,63 \%$

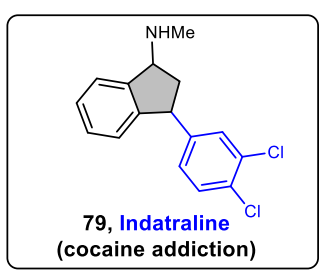

(cocaine addiction)

Phenylacetylene (4)

80<smiles>CCOC(=O)C1C(=O)c2cc(OCCNCC3C(=O)c4cc(O[GaH2])ccc4C3c3ccccc3)ccc2C1c1ccccc1</smiles>

82

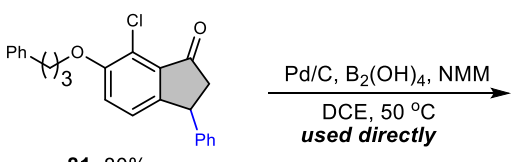

83, 66\% (two steps)

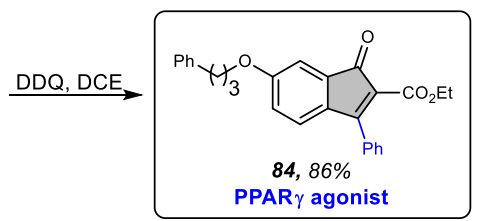

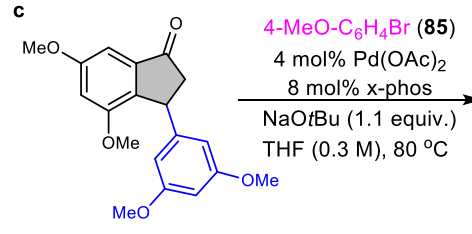

69

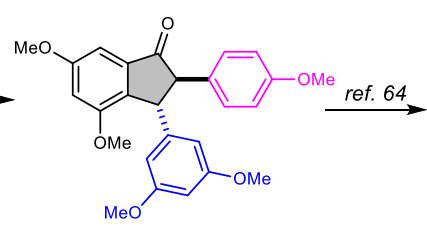

$86,82 \%,>20 / 1 d r$

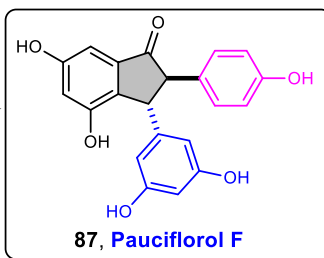

87, Pauciflorol F

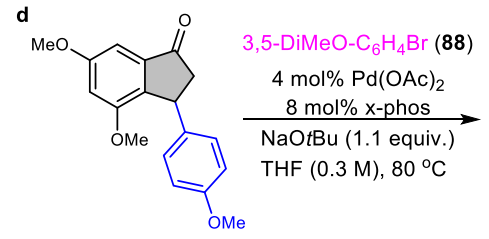

70

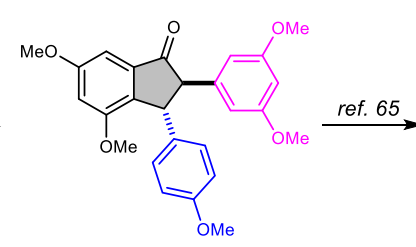

89, $87 \%,>20 / 1 d r$

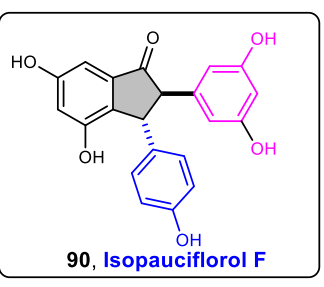

Fig. 5 Synthetic applications of indanones. a Key intermediate for indatraline construction could be afforded by the developed protocol. b Synthesis of PPAR $\gamma$ agonist using this strategy. NMM 4-methylmorpholine. c Powerful protocol to prepare pauciflorol F. d Formal synthesis Isopauciflorol F.

that the carbonyl group plays a key role during the cyclization process to provide a five-membered ring product. We next tested the competition of aryl $\mathrm{C}-\mathrm{H}$ bonds and weaker $\mathrm{Cs} p^{3}-\mathrm{H}$ bond involved in the intermediate. In these cases, the HAT between vinyl radical and weaker $\mathrm{C}-\mathrm{H}$ bonds outcompetes watermediated pathways affording the furan-derived products with little indanones detected (101 vs. 102,104 vs. 105,107 vs. 108). These phenomena not only showed obvious chemo-selectivity in 


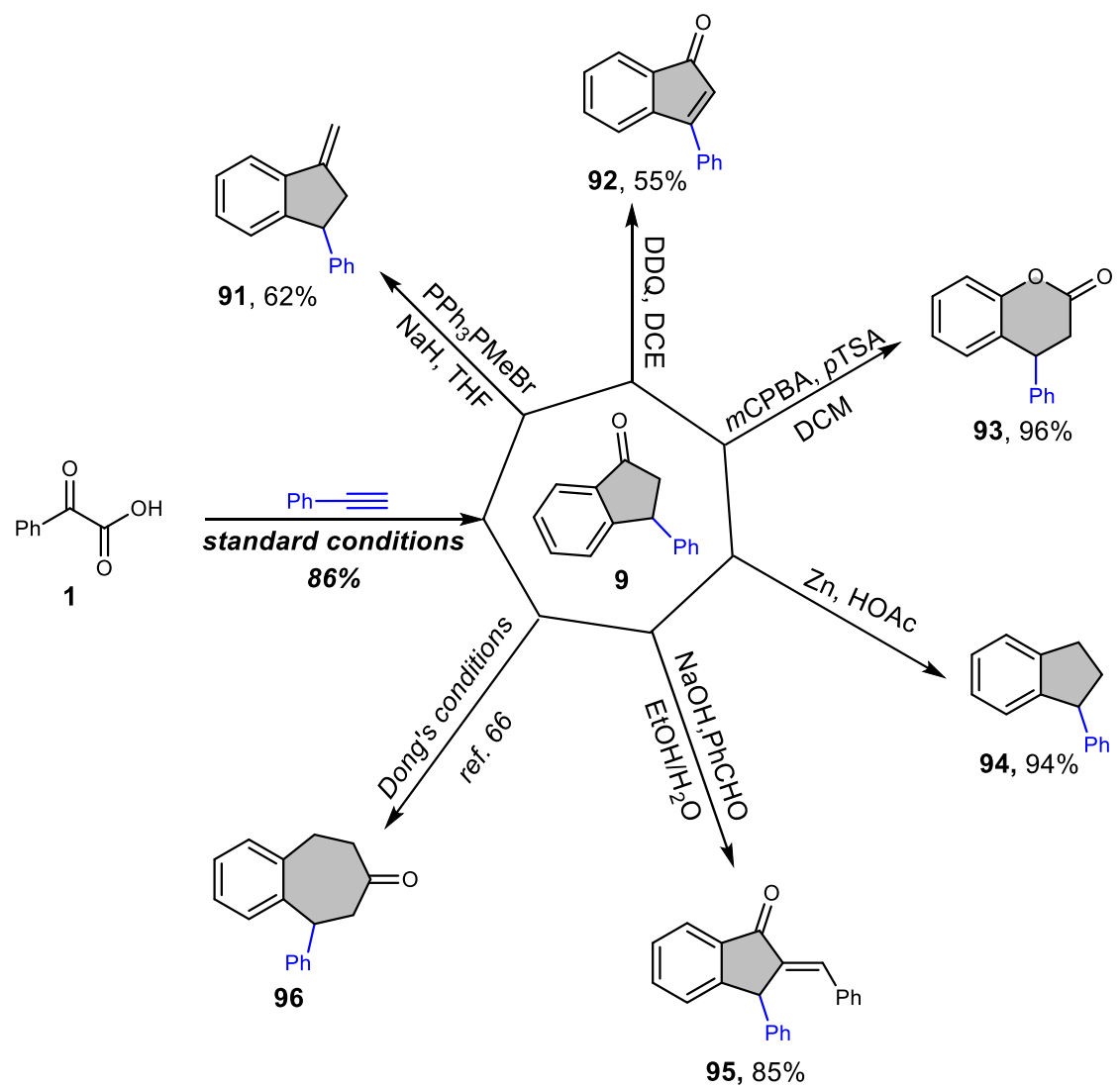

Fig. 6 Further transformations of indanones. The indanone $\mathbf{9}$ was prepared from $\mathbf{1}$ under standard conditions. The indanone $\mathbf{9}$ was efficiently transformed to diverse compounds, such as alkene $\mathbf{9 1}$, indenone $\mathbf{9 2}$, lactone $\mathbf{9 3}$, 1-phenyl-2,3-dihydro-1H-indene $\mathbf{9 4}$, (E)-2-benzylidene-3-phenyl-2,3-dihydro-1H-inden1-one $\mathbf{9 5}$, and benzocycloheptenone $\mathbf{9 6}$, respectively.

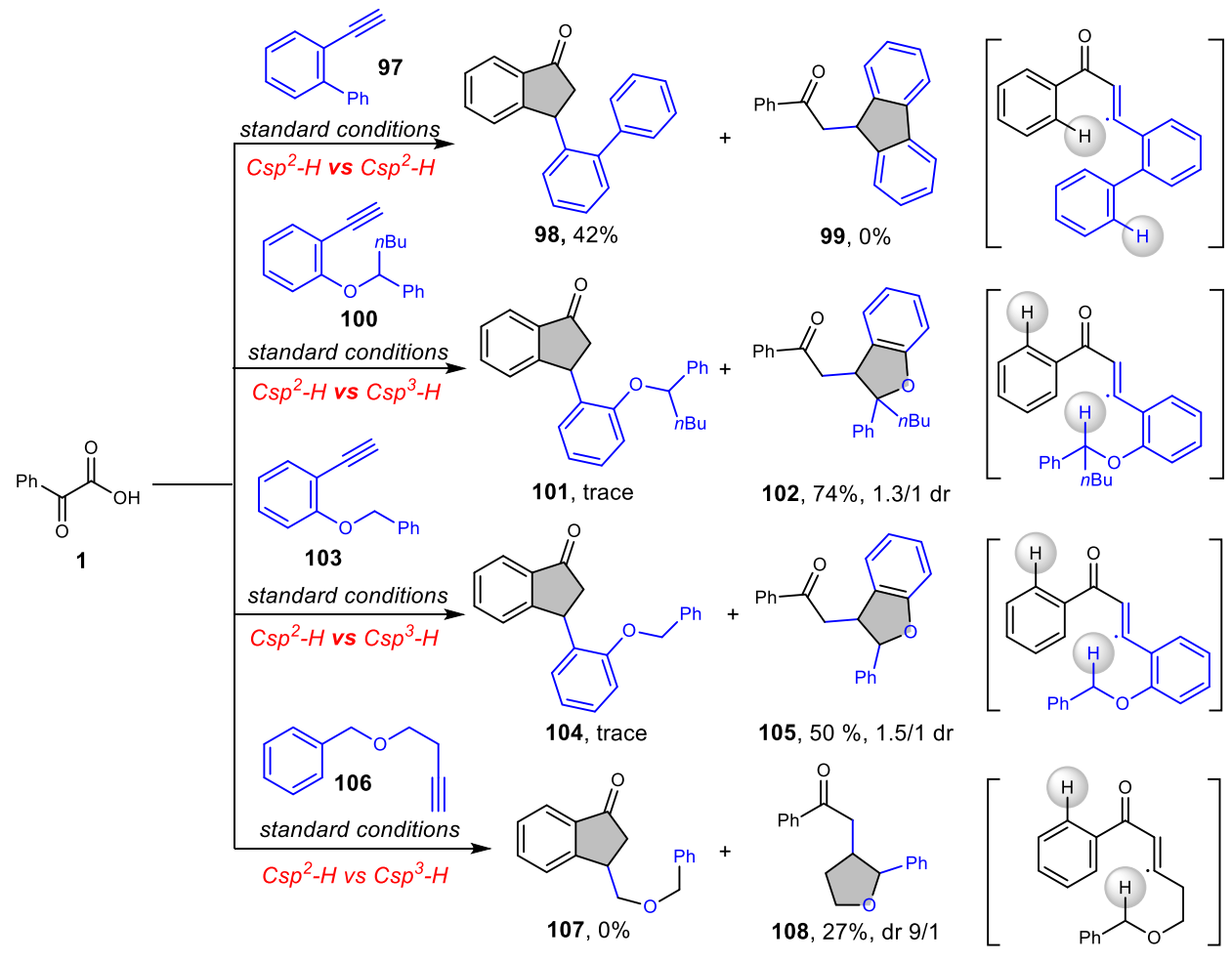

Fig. 7 Mechanistic probes for chemo- and regio-selectivity. Competitions between different $\mathrm{C}-\mathrm{H}$ bonds. The indanone $\mathbf{9 8}$ was formed in the presence of another $\mathrm{Csp}^{2}-\mathrm{H}$ bond. Only furan-derived products $\left(\mathbf{1 0 2}, \mathbf{1 0 5}\right.$ and 108) could be isolated when weaker $\mathrm{Csp} \mathrm{P}^{3}-\mathrm{H}$ bonds have existed. 


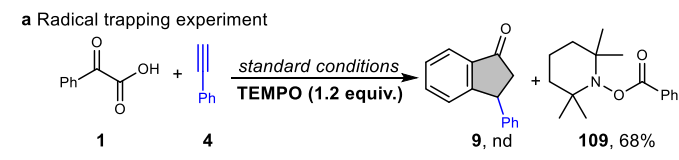

c Deuteration and kinetic isotope effect experiments
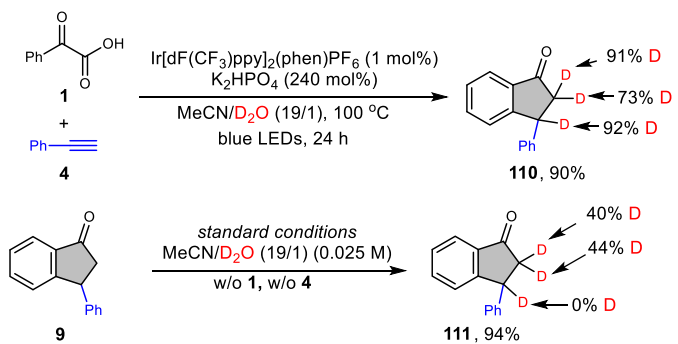

9 $111,94 \%$
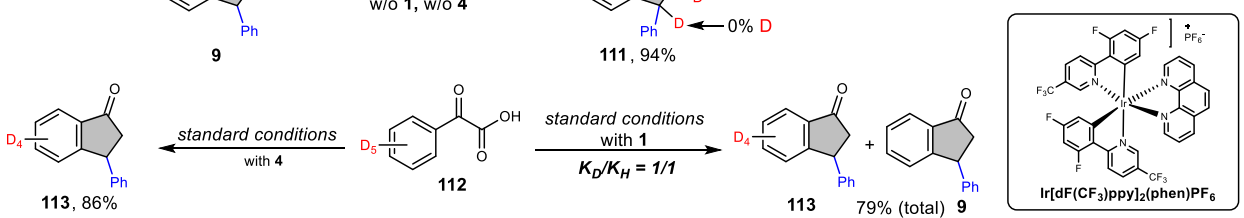

d Exploration of possible intermediate involved
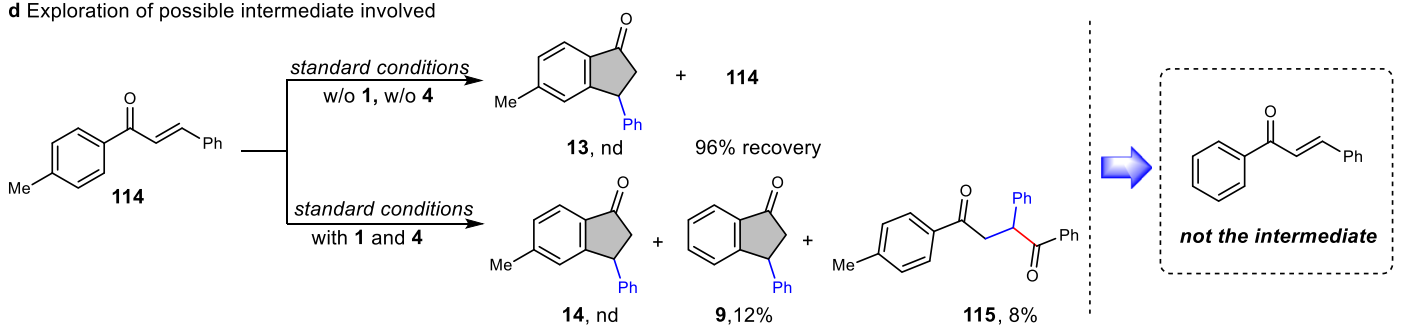

Fig. 8 Mechanistic studies. a Radical-trapping experiment with 2,2,6,6-tetramethylpiperidinooxy (TEMPO). b Light-on-off the experiment. c Deuterated and kinetic isotope experiments. $\mathbf{d}$ Exploration of possible intermediate involved.

the presence of weaker $\mathrm{C}-\mathrm{H}$ bonds but also deliver strong evidence for the existence of vinyl radical. Moreover, the deuterium substitution experiment with $\mathrm{D}_{2} \mathrm{O}$ using substrate 100 was performed and found that no deuterated benzylic product was formed (see Supplementary Fig. 1 for details). The result indicated that the corresponding product was formed through an intramolecular 1,5-HAT followed by a Giese addition, which is not a water-mediated pathway.

To better understand the detailed mechanism of the reaction, a series of mechanistic studies were performed (Fig. 8). In the presence of radical trap TEMPO, the reaction was completely shut down (Fig. 8a), indicating that a radical intermediate might be involved in this transformation. More importantly, acyl-trapped product, 2,2,6,6-tetramethylpiperidin-1-yl benzoate 109 was isolated in high yield, further supporting the reaction proceeds through a radical decarboxylation pathway and the intermediacy of an acyl radical. The light-on-off experiment demonstrates the radical chain mechanism is less likely involved (Fig. 8b). To verify if $\mathrm{H}_{2} \mathrm{O}$ was involved in the reaction as proposed, $\mathrm{D}_{2} \mathrm{O}$ was used and subjected to the optimal reaction conditions, deuterated product $\mathbf{1 1 0}$ was observed in $90 \%$ yield when $\mathrm{D}_{2} \mathrm{O}$ was utilized in place of $\mathrm{H}_{2} \mathrm{O}$, demonstrating the benzylic site of hydrogen is originated from water (Fig. 8c, top). To further test whether deuterated product 110 was generated from the indanone $\mathbf{9}$ under standard conditions through $\mathrm{H} / \mathrm{D}$ exchange with $\mathrm{D}_{2} \mathrm{O}$, indanone 9 was subjected to the reaction conditions with $\mathrm{D}_{2} \mathrm{O}$ instead of $\mathrm{H}_{2} \mathrm{O}$ (Fig. $8 \mathrm{c}$, middle). Interestingly, a deuterated indanone 111 was formed, in which only the $\mathrm{CH}_{2}$ of indanone was deuterated through $\mathrm{H} / \mathrm{D}$ exchange with $\mathrm{D}_{2} \mathrm{O}$ and no deuterated benzylic product was formed. This experiment suggested that the benzylic $\mathrm{C}-\mathrm{H}$ cannot be deuterated through $\mathrm{H} / \mathrm{D}$ exchange with $\mathrm{D}_{2} \mathrm{O}$ under standard conditions. To verify whether the 1,5-
HAT is involved in the reaction, deuterated phenylglyoxylic acid $\mathbf{1 1 2}$ was used as substrate under standard conditions (Fig. 8c, bottom, left). The absolute indanone $\mathbf{1 1 3}$ without deuterium transfer was obtained in $86 \%$ yield, demonstrating that the reaction did not proceed via 1,5 -HAT pathway. In addition, it also indicated that no obvious direct hydrogen transfer occurred from the aryl position to the corresponding methylene and benzylic site of indanone, which is not in line with the 1,2- and 1,3-HAT. Taken together, the hydrogen atom of benzylic $\mathrm{C}-\mathrm{H}$ of indanone should come from water during the catalytic reaction process, neither from the aryl $\mathrm{C}-\mathrm{H}$ via $1,5-$ HAT nor from water through H/D exchange after reaction completion. Kinetic isotope experiments (KIE) were also performed to have more insight into the reaction mechanism (Fig. $8 \mathrm{c}$, bottom, right). Since no obvious KIE effect $\left(K_{\mathrm{D}} / K_{\mathrm{H}}=1 / 1\right)$ was detected when the equivalent of $\mathbf{1}$ and $\mathbf{1 1 2}$ were subjected to the reaction conditions with alkyne 4 , according to the intermolecular competition experiment, aryl $\mathrm{C}-\mathrm{H}$ bond cleavage was not likely the ratedetermining step. Additionally, when ketone 114 was performed under standard conditions, no cyclized product 13 was detected but with a recovery of $\mathbf{1 1 4}$ in $96 \%$ (Fig. 8d, top). Furthermore, when chalcone $\mathbf{1 1 4}$ was added as an additive to the model reaction, only the predictable indanone 9 and Giese-type reaction product 115 could be monitored (Fig. 8d, bottom). These control experiments strongly indicated that the chalcone $\mathbf{1 1 4}$ is less likely the intermediate involved in the reaction.

DFT calculation was subsequently employed to provide further insight into the mechanism of this decarboxylative annulation reaction (Fig. 9a). According to the computational calculations, an intermolecular radical addition with phenylacetylene 4 takes place via transition state TS1 with a free energy barrier of $23.7 \mathrm{kcal} / \mathrm{mol}$ to afford the vinyl radical 5 . Then an intramolecular radical addition 


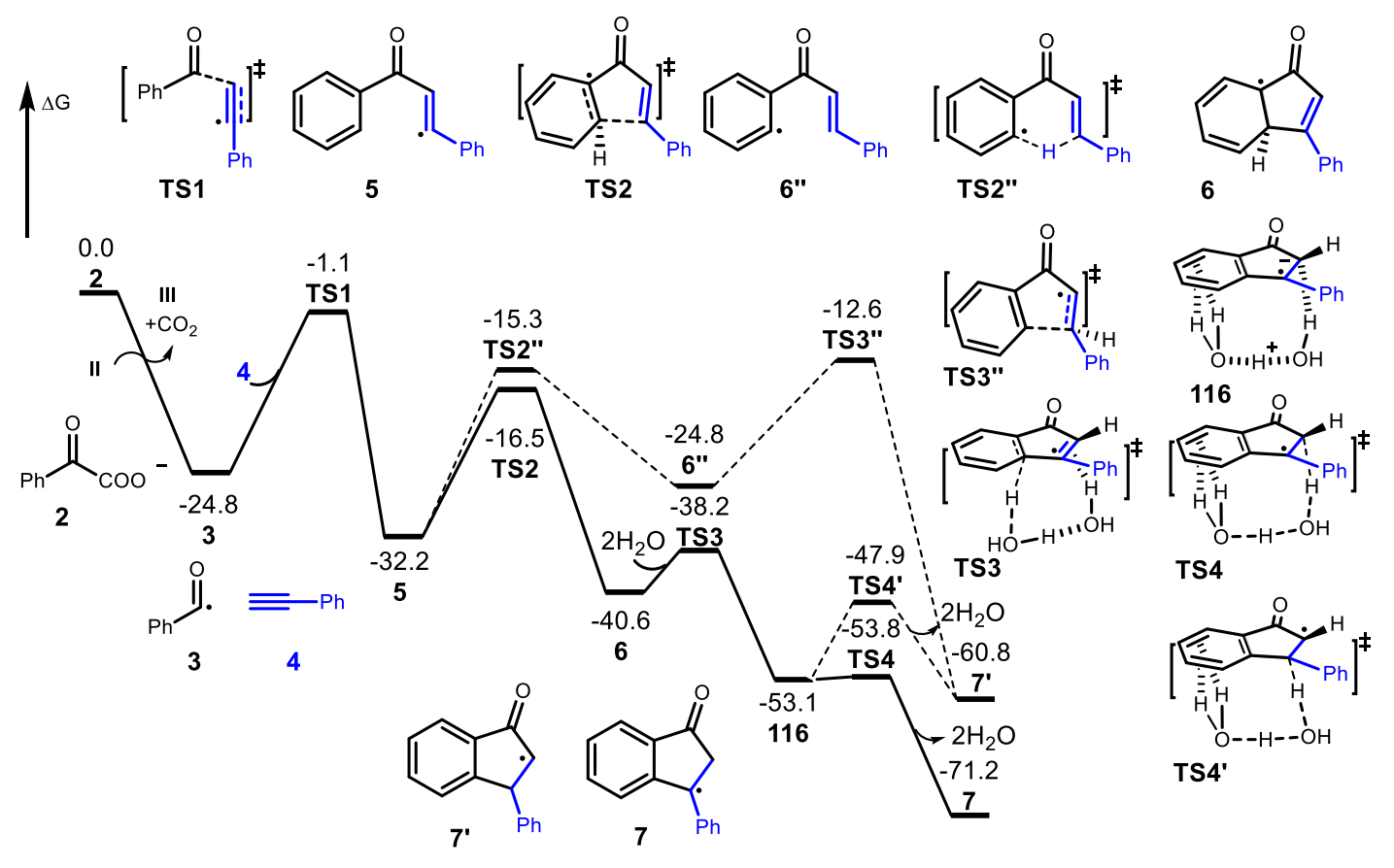

b
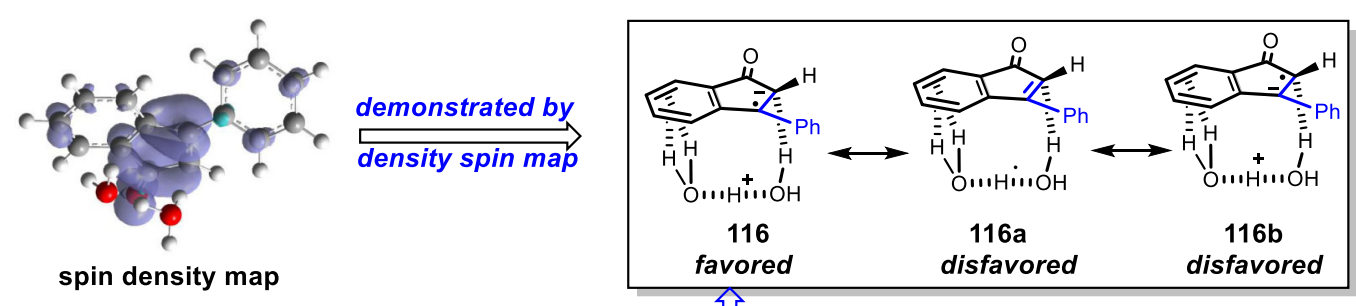

spin density map

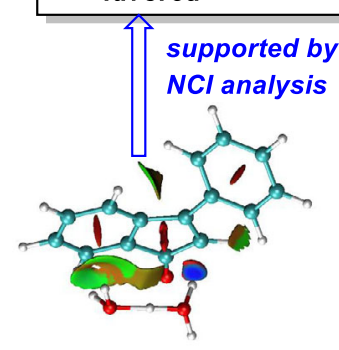

$\mathrm{NCl}$ analysis

Fig. 9 The free energy barrier for the reaction and the spin density and noncovalent interaction $(\mathrm{NCl})$ analysis for the intermediate 116. a Computational studies. $\mathbf{b}$ The spin density map and $\mathrm{NCl}$ analysis for the intermediate $\mathbf{1 1 6 .}$

would occur via transition state TS2 with a free energy barrier of $15.7 \mathrm{kcal} / \mathrm{mol}$ to achieve annulation and form a dearomatized intermediate 6. Because of the similar BDE values of $\mathrm{C}-\mathrm{H}$ bonds between alkenes and arenes, we considered an alternative intramolecular 1,5-hydrogen shift theoretically via transition state TS2". However, the relative free energy of generated phenyl radical $6^{\prime \prime}$ is $7.4 \mathrm{kcal} / \mathrm{mol}$ higher than that of vinyl radical 5. Moreover, the relative free energy for the corresponding annulation transition state TS3 $^{\prime \prime}$ is also $3.9 \mathrm{kcal} / \mathrm{mol}$ higher than that of TS2. This analysis revealed that the generation of intermediate $\mathbf{6}$ is a favorable pathway. These calculations were highly consistent with our experimental observations (Fig. 8c). Next, we focused on understanding the exact role of water in the formation of intermediate 7 or $7^{\prime}$. When dearomatized intermediate $\mathbf{6}$ is formed, from calculations, a waterassisted stepwise 1,3-hydrogen transfer would provide a more stable benzylic radical 7 with rearomatization. In this process, two water molecules were used to achieve dehydrogenation via transition state
TS3 to afford a complex radical intermediate $\mathbf{1 1 6}$ with a free energy barrier of only $2.4 \mathrm{kcal} / \mathrm{mol}$ (see Supplementary Fig. 9 for other water molecules assisted pathway). Two other possible resonance structures of intermediate $\mathbf{1 1 6}$ could be drawn as electron-neutral indenone with hydrated hydrogen radical 116a and zwitterionic in indenolate radical with protonated water $\mathbf{1 1 6 b}$. The spin density map of intermediate $\mathbf{1 1 6}$ clearly revealed that spin density is majorly located at indenone moiety. Meanwhile, the electrostatic potential map also exhibits a charge-separated character (Fig. 9b). Therefore, zwitterionic resonance structure $\mathbf{1 1 6}$ has a more appreciable contribution for this intermediate. Interestingly, noncovalent interaction (NCI) analysis of intermediate $\mathbf{1 1 6}$ also revealed a strong hydroxyl- $\pi$ interaction between indenolate radical and hydrated proton, which explained the stability of this intermediate. When intermediate $\mathbf{1 1 6}$ is formed, a rapid protonation takes place via transition state TS4 resulting in the formation of benzylic radical 7 with the release of two water molecules (see Supplementary Data 1 
for the coordination of all the structures involved in the computational calculations). Therefore, as we designed, water acts as a hydrogen radical-shuttle catalyst, promoting the favorable 1,3hydrogen transfer with the formation of intermediate 7 .

In summary, we have developed a decarboxylative annulation for indanones synthesis via photoredox/HAT catalysis with water as hydrogen radical-shuttle (HRS). This protocol provides a powerful platform to construct indanones with broad substrate scope, excellent functional group tolerance, internal hydrogen radical transfer, atomand step-economy, using simple and available 2-oxo-2-phenylacetic acids and readily available alkynes. Moreover, the exact role of water in this developed strategy was demonstrated by mechanistic experiments and DFT calculations, as we designed, facilitating the hydrogen transfer and acting as the hydrogen source. Namely, acting as a HRS catalyst was critical to the success of this process. Additionally, the key intermediate $\mathbf{1 1 6}$ was further demonstrated by spin density map and NCI analysis. Most importantly, to the best of our knowledge, this system provides an aromatization model, representing a breakthrough in hydrogen radical transfer assisted by HRS. This hydrogen transfer is mechanistically distinctive from that of typical PS-promoted, providing a complementary process in hydrogen transfer and a feasible solution in achieving 1,2-, or 1,3HAT. We expect this strategy could be widely adopted and further promote the development of direct functionalization of aryl Csp $p^{2}-\mathrm{H}$ via HRS-assisted hydrogen transfer.

\section{Methods}

Materials. Unless otherwise noted, all the materials were obtained commercially and used without further purification. All the solvents were treated according to general methods. Flash column chromatography was performed over silica gel (300-400 mesh). See Supplementary Methods for experimental details.

General procedures for the indanones synthesis. To an oven-dried $50 \mathrm{~mL}$ flask, $\operatorname{Ir}\left[\mathrm{dF}\left(\mathrm{CF}_{3}\right) \mathrm{ppy}_{2}\right.$ (phen) $\mathrm{PF}_{6}(0.005 \mathrm{mmol}), 2$-aryl-2-oxocarboxylic acid $(1.0 \mathrm{mmol})$ and $\mathrm{K}_{2} \mathrm{HPO}_{4}(1.2 \mathrm{mmol})$ were added sequentially under $\mathrm{N}_{2}$. The flask was evacuated and back-filled with $\mathrm{N}_{2}$ for three times, then alkyne $(0.5 \mathrm{mmol}), \mathrm{H}_{2} \mathrm{O}(1 \mathrm{~mL})$, and $\mathrm{MeCN}(19 \mathrm{~mL})$ was added. The reaction mixture was irradiated by $12 \mathrm{~W}$ blue LEDs at a distance of $5 \mathrm{~cm}$ for $24 \mathrm{~h}$ at $100{ }^{\circ} \mathrm{C}$. The reaction mixture was cooled to $\mathrm{rt}$ and filtered through a short pad of silica using ethyl acetate. The filtrate was concentrated in vacuo before it was purified by flash chromatography on silica gel to afford the desired indanone product.

Computational method. All the calculations in this study were performed using the Gaussian 16 program package. ${ }^{67}$ The All the geometries were optimized at the M06-2X $\mathrm{X}^{68} / 6-31 \mathrm{G}(d, p)$ and SDD for Ir level, and the solvent effect was utilized the polarizable continuum model using integral equation formalism model (IEFPCM) in hexane solvent. ${ }^{69}$ All the optimized stationary points had been identified as minima (zero imaginary frequencies) and transition states (one imaginary frequency), via the vibrational analysis. The solution-translational entropy correction has been calculated with the THERMO program. ${ }^{70}$

\section{Data availability}

The authors declare that the data relating to the characterization of materials and products, general methods, optimization studies, experimental procedures, mechanistic studies, HRMS data and NMR spectra, computational studies are available within the article and its Supplementary Information as well as supplementary data.

Received: 27 May 2021; Accepted: 19 August 2021;

Published online: 06 September 2021

\section{References}

1. Mayer, J. M. Understanding hydrogen atom transfer: from bond strengths to Marcus theory. Acc. Chem. Res. 44, 36-46 (2011).

2. Chen, M. S. \& White, M. C. Combined effects on selectivity in Fe-catalyzed methylene oxidation. Science 327, 566-571 (2010).

3. Newhouse, T. \& Baran, P. S. If C-H bonds could talk: selective C-H bond oxidation. Angew. Chem. Int. Ed. 50, 3362-3374 (2011).
4. Liu, W. et al. Oxidative aliphatic $\mathrm{C}-\mathrm{H}$ fluorination with fluroide ion catalyzed by a manganese porphyrin. Science 337, 1322-1325 (2012).

5. Jeffrey, J. L., Terrett, J. A. \& MacMillan, D. W. C. O-H hydrogen bonding promotes $\mathrm{H}$-atom transfer from a $\mathrm{C}-\mathrm{H}$ bonds for $\mathrm{C}$-alkylation of alcohols. Science 349, 1532-1536 (2015).

6. Choil, G. J., Zhu, Q., Miller, D. C., Gu, C. J. \& Knowles, R. R. Catalytic alkylation of remote $\mathrm{C}-\mathrm{H}$ bonds enabled by proton-coupled electron transfer. Nature 539, 268-271 (2016).

7. Chu, J. C. K. \& Rovis, T. Amide-directed photoredox-catalysed C-C bond formation at unactivated $s p^{3} \mathrm{C}-\mathrm{H}$ bonds. Nature 539, 272-275 (2016).

8. Hu, X.-Q., Chen, J.-R. \& Xiao, W.-J. Controllable remote C-H bond functionalization by visible-light photocatalysis. Angew. Chem. Int. Ed. 56, 1960-1962 (2017).

9. Chu, J. C. K. \& Rovis, T. Complementary strategies for directed C $\left(s p^{3}\right)-\mathrm{H}$ functionalization: a comparison of transition-metal-catalyzed activation, hydrogen atom transfer, and carbene/nitrene transfer. Angew. Chem. Int. Ed. 57, 62-101 (2018)

10. Robertson, J., Pillai, J. \& Lush, R. K. Radical translocation reactions in synthesis. Chem. Soc. Rev. 30, 94-103 (2001).

11. Sarkar, S., Cheung, K. P. S. \& Gevorgyan, V. C-H functionalization reactions enabled by hydrogen atom transfer to carbon-centered radicals. Chem. Sci. 11, 12974-12993 (2020).

12. Xia, Y., Wang, L. \& Studer, A. Site-selective remote radical C-H functionalization of unactivated $\mathrm{C}-\mathrm{H}$ bonds in amides using sulfone reagents. Angew. Chem. Int. Ed. 57, 12940-12944 (2018).

13. Lafrance, M. \& Fagnou, K. Palladium-catalyzed benzene arylation: incorporation of catalytic pivalic acid as a proton shuttle and a key element in catalyst design. J. Am. Chem. Soc. 128, 16496-16497 (2006).

14. Davies, D. L., Macgregor, S. A. \& McMullin, C. L. Computational studies of carboxylate-assisted $\mathrm{C}-\mathrm{H}$ activation and functionalization at group 8-10 transition metal centers. Chem. Rev. 117, 8649-8709 (2017).

15. Ackermann, L. Carboxylate-assisted transition-metal-catalyzed $\mathrm{C}-\mathrm{H}$ bond functionalizations: mechanism and scope. Chem. Rev. 111, 1315-1345 (2011).

16. Liang, Y., Zhou, H.-L. \& Yu, Z.-X. Why is copper(I) complex more competent than dirhodium(II) complex in catalytic asymmetric $\mathrm{O}-\mathrm{H}$ insertion reactions? A computational study of the metal carbenoid $\mathrm{O}-\mathrm{H}$ insertion into water. J. Am. Chem. Soc. 131, 17783-17785 (2009).

17. Xu, B., Zhu, S.-F., Xie, X.-L., Shen, J.-J. \& Zhou, Q.-L. Asymmetric N-H insertion reaction cooperatively catalyzed by rhodium and chiral spiro phosphoric acids. Angew. Chem. Int. Ed. 50, 11483-11486 (2011)

18. $\mathrm{Xu}, \mathrm{B}$. et al. Highly enantioselective $\mathrm{S}-\mathrm{H}$ bond insertion cooperatively catalyzed by dirhodium complexes and chiral spiro phosphoric acids. Chem. Sci. 5, 1442-1448 (2014).

19. Xu, B., Li, M.-L., Zuo, X.-D., Zhu, S.-F. \& Zhou, Q.-L. Catalytic asymmetric arylation of $\alpha$-aryl- $\alpha$-diazoacetates with aniline derivatives. J. Am. Chem. Soc. 137, 8700-8703 (2015).

20. Guo, J.-X., Zhou, T., Xu, B., Zhu, S.-F. \& Zhou, Q.-L. Enantioselective synthesis of $\alpha$-alkenyl $\alpha$-amino acids via $\mathrm{N}-\mathrm{H}$ insertion reactions. Chem. Sci. 7, 1104-1108 (2016).

21. Wang, G.-P., Chen, M.-Q., Zhu, S.-F. \& Zhou, Q.-L. Enantioselective Nazarov cyclization of indole enones cooperatively catalyzed by Lewis acids and chiral Brønsted acids. Chem. Sci. 8, 7197-7202 (2017).

22. Ren, Y.-Y., Zhu, S.-F. \& Zhou, Q.-L. Chiral Proton-transfer shuttle catalysts for carbene insertion reactions. Org. Biomol. Chem. 16, 3087-3094 (2018).

23. Li, Y.-P. et al. Chiral spiro phosphoric acid-catalyzed friedel-crafts conjugate addition/enantioselective protonation reactions. ACS Catal. 9, 6522-6529 (2019).

24. Xue, J., Luk, H. L. \& Platz, M. S. Direct observation of a carbene-alcohol ylide. J. Am. Chem. Soc. 133, 1763-1765 (2011).

25. Miller, D. C., Choi, G. J., Orbe, H. S. \& Knowles, R. R. Catalytic olefin hydroamidation enabled by proton-coupled electron transfer. J. Am. Chem. Soc. 137, 13492-13495 (2015)

26. Topczewski, J. J., Cabrera, P. J., Saper, N. I. \& Sanford, M. S. Palladiumcatalysed transannular $\mathrm{C}-\mathrm{H}$ functionalization of alicyclic amines. Nature 531, 220-224 (2016)

27. Fujiwara, Y. et al. Practical and innate carbon-hydrogen functionalization of heterocycles. Nature 492, 95-99 (2012).

28. Wolff, M. E. Cyclization of N-halogenated amines (The Hofmann-Loffler reaction). Chem. Rev. 63, 55-64 (1963).

29. Roberts, B. P. Polarity-reversal catalysis of hydrogen-atom abstraction reactions: concepts and applications in organic chemistry. Chem. Soc. Rev. 28, 25-35 (1999)

30. Le, C., Liang, Y., Evans, R. W., Li, X. \& MacMillan, D. W. C. Selective $s p^{3}$ C-H alkylation via polarity-match-based cross-coupling. Nature 547, 79-83 (2017).

31. Keylor, M. H., Matsuura, B. S. \& Stephenson, C. R. Chemistry and biology of resveratrol-derived natural products. Chem. Rev. 115, 8976-9027 (2015).

32. Borie, C., Ackermann, L. \& Nechab, M. Enantioselective syntheses of indanes: from organocatalysis to C-H functionalization. Chem. Soc. Rev. 45, 1368-1386 (2016). 
33. Turek, M., Szczęsna, D., Koprowski, M. \& Bałczewski, P. Synthesis of 1-indanones with a broad range of biological activity. Beilstein J. Org. Chem. 13, 451-494 (2017)

34. Sugimoto, H., Yamanish, Y., Limura, Y. \& Kawakami, Y. Donepezil hydrochloride (E2020) and other acetylcholinesterase inhibitors. Curr. Med. Chem. 7, 303-339 (2000).

35. Kuroyanagi, M., Fukuoka, M., Yoshihira, K. \& Natori, S. The absolute configurations of Pterosins, 1-indanone derivatives from bracken, pteridium aquilinum var. latiusculum. Chem. Pharm. Bull. 22, 723-726 (1974).

36. Vasilyev, A. V. et al. Chemistry of 1,3-diarylpropynones in superacids. Org. Biomol. Chem. 2, 3483-3849 (2004).

37. Yan, X., Zhou, S., Zhao, P. \& Xi, C. MeOTf-induced carboannulation of arylnitriles and aromatic alkynes: a new metal-free strategy to construct indenones. Chem. Commun. 50, 2775-2777 (2014).

38. Yue, G., Lei, K., Hirao, H. \& Zhou, J. Palladium-catalyzed asymmetric reductive heck reaction of aryl halides. Angew. Chem. Int. Ed. 54, 6531-6535 (2015).

39. Pan, C., Huang, B., Hu, W., Feng, X. \& Yu, J. Metal-free radical oxidative annulation of ynones with alkanes to access indenones. J. Org. Chem. 81, 2087-2093 (2016).

40. Zhang, X.-S., Jiao, J.-Y., Zhang, X.-H., Hu, B.-L. \& Zhang, X.-G. Synthesis of 2 -sulfenylindenones via one-pot tandem meyer-schuster rearrangement and radical cyclization of arylpropynols with disulfides. J. Org. Chem. 81, 5710-5716 (2016).

41. Liebeskind, L. S. \& South, M. S. Reaction of $o$-diiodobenzene with alkynes and nickel carbonyl. Synthesis of substituted indenones. J. Org. Chem. 45, 5426-5429 (1980).

42. Larock, R. C., Tian, Q. \& Pletnev, A. A. carbocycle synthesis via carbopalladation of nitriles. J. Am. Chem. Soc. 121, 3238-3239 (1999).

43. Miura, T. \& Murakami, M. Rhodium-catalyzed annulation reactions of 2-cyanophenylboronic acid with alkynes and strained alkenes. Org. Lett. 7, 3339-3341 (2005).

44. Tsukamoto, H. \& Kondo, Y. Palladium(II)-catalyzed annulation of alkynes with ortho-ester-containing phenylboronic acids. Org. Lett. 9, 4227-4230 (2007).

45. Morimoto, T. et al. Rh(I)-catalyzed CO gas-free carbonylative cyclization reactions of alkynes with 2-bromophenylboronic acids using formaldehyde. Org. Lett. 11, 1777-1780 (2009).

46. Quan, L. G., Gevorgyan, V. \& Yamamoto, Y. Intramolecular nucleophilic addition of vinylpalladiums to aryl ketones. J. Am. Chem. Soc. 121, 3545-3546 (1999).

47. Chang, K.-J., Rayabarapu, D. K. \& Cheng, C.-H. Cobalt-catalyzed carbocyclization of $o$-iodobenzaldehydes and $o$-iodophenylketones with alkynes. Org. Lett. 5, 3963-3966 (2003).

48. Rayabarapu, D. K. \& Cheng, C.-H. Nickel-catalyzed regioselective carbocyclization of ortho-halophenyl ketones with propiolates: an efficient route to disubstituted indenols. Chem. Commun. 942-943 (2002).

49. Chen, Y., Ding, Z., Wang, Y., Liu, W. \& Kong, W. Synthesis of indanones and spiroindanones by diastereoselective annulation based on a hydrogen autotransfer strategy. Angew. Chem. Int. Ed. 60, 5273-5278 (2021).

50. Harada, Y. et al. Rh(I)-catalyzed carbonylative cyclization reactions of alkynes with 2-bromophenylboronic acids leading to indenones. J. Am. Chem. Soc. 129, 5766-5771 (2007).

51. Kuninobu, Y., Matsuki, T. \& Takai, K. Rhenium-catalyzed synthesis of indenones by novel dehydrative trimerization of aryl aldehydes via $\mathrm{C}-\mathrm{H}$ bond activation. Org. Lett. 12, 2948-2950 (2010).

52. Li, B.-J., Wang, H.-Y., Zhu, Q.-L. \& Shi, Z.-J. Rhodium/copper-catalyzed annulation of benzimides with internal alkynes: indenone synthesis through sequential C-H and C-N Cleavage. Angew. Chem. Int. Ed. 51, 3948-3952 (2012).

53. Prier, C. K., Rankic, D. A. \& MacMillan, D. W. C. Visible light photoredox catalysis with transition metal complexes: applications in organic synthesis. Chem. Rev. 113, 5322-5363 (2013).

54. Narayanam, C. R. J. \& Stephenson, C. R. J. Visible light photoredox catalysis: applications in organic synthesis. Chem. Soc. Rev. 40, 102-113 (2011).

55. Raviola, C., Protti, S., Ravelli, D. \& Fagnoni, M. Photogenerated acyl/ alkoxycarbonyl/carbamoyl radicals for sustainable synthesis. Green Chem. 21, 748-764 (2019).

56. Lei, T. et al. General and efficient intermolecular [2+2] photodimerization of chalcones and cinnamic acid derivatives in solution through visible light catalysis. Angew. Chem. Int. Ed. 56, 15407-15410 (2017).

57. Wang, G.-Z., Shang, R., Cheng, W.-M. \& Fu, Y. Decarboxylative 1,4-addition of $\alpha$-oxocarboxylic acids with Michael acceptors enabled by photoredox catalysis. Org. Lett. 17, 4830-4833 (2015)

58. Wille, U. Radical cascades Initiated by intermolecular radical addition to alkynes and related triple bond systems. Chem. Rev. 113, 813-853 (2013)

59. Giese, B. \& Lachhein, S. Addition of alkyl radicals to alkynes: distinction between radical and ionic nucleophiles. Angew. Chem. Int. Ed. Engl. 21, 768-775 (1982).

60. Brooks, M. A. \& Scott, L. T. 1,2-Shifts of hydrogen atoms in aryl radicals. J. Am. Chem. Soc. 121, 5444-5449 (1999).
61. Clark, T. 1,3-Hydrogen shifts in olefin radical cations: an ab Initio study. J. Am. Chem. Soc. 109, 6838-6840 (1987).

62. Friomowitz, M. et al. Slow-onset, long-duration 3-(3',4'-dichlorophenyl)-1indanamine monoamine reuptake blockers as potential medications to treat cocaine abuse. J. Med. Chem. 43, 4981-4992 (2000).

63. Ahn, J. H. et al. Indenone derivatives: a novel template for peroxisome proliferator-activated receptor $\gamma(\mathrm{PPAR} \gamma)$ agonists. J. Med. Chem. 49, 4781-4784 (2006)

64. Snyder, S. A., Zografos, A. L. \& Lin, Y. Total synthesis of resveratrol-based natural products: a chemoselective solution. Angew. Chem. Int. Ed. 46, 8186-8191 (2007)

65. Tang, M.-L. et al. Sulfoxide-based enantioselective Nazarov cyclization: divergent syntheses of $(+)$-Isopaucifloral F, (+)-Quadrangularin A, and (+)-Pallidol. Chem. Eur. J. 22, 14535-14539 (2016).

66. Xia, Y., Ochi, S. \& Dong, G. Two-carbon ring expansion of 1-indanones via insertion of ethylene into carbon-carbon bonds. J. Am. Chem. Soc. 141, 13038-13042 (2019)

67. Frisch, M. J. et al. Gaussian16 (Gaussian, Inc., 2016).

68. Zhao, Y. \& Truhlar, D. G. The M06 suite of density functionals for main group thermochemistry, thermochemical kinetics, noncovalent interactions, excited states, and transition elements: two new functionals and systematic testing of four M06-class functionals and 12 other functionals. Theor. Chem. Acc. 120, 215-241 (2008).

69. Scalmani, G. \& Frisch, M. J. Continuous surface charge polarizable continuum models of solvation. I. General formalism. J. Chem. Phys. 132, 114110 (2010).

70. Fang, D. -C. THERMO (Beijing Normal University, 2013).

\section{Acknowledgements}

We are grateful for the financial support from the National Natural Science Foundation of China (22071062, 22001079, 21871096), the Ministry of Science and Technology of the People's Republic of China (2016YFA0602900), Guangdong Science and Technology Department (2018B030308007), and the China Postdoctoral Science Foundation (2020M682694). We also thank Dr. Tongxiang Cao from South China University of Technology for assistance with melting points determination.

\section{Author contributions}

B.Y. and S.Z. designed the experiments. B.Y. performed experiments. S.-J.L. and Y.L. performed DFT calculations and wrote the paper's DFT calculations. S.Z. and Y.W. codirected the synthetic applications of indanones. B.Y. wrote the paper and S.Z. revised reviewed \& edited the paper. All authors discussed the results and commented on the manuscript. S.Z. directed the whole project.

\section{Competing interests}

The authors declare no competing interests.

\section{Additional information}

Supplementary information The online version contains supplementary material available at https://doi.org/10.1038/s41467-021-25594-4.

Correspondence and requests for materials should be addressed to Y.W., Y.L. or S.Z.

Peer review information Nature Communications thanks the anonymous reviewer(s) for their contribution to the peer review of this work

Reprints and permission information is available at http://www.nature.com/reprints

Publisher's note Springer Nature remains neutral with regard to jurisdictional claims in published maps and institutional affiliations.

Open Access This article is licensed under a Creative Commons Attribution 4.0 International License, which permits use, sharing, adaptation, distribution and reproduction in any medium or format, as long as you give appropriate credit to the original author(s) and the source, provide a link to the Creative Commons license, and indicate if changes were made. The images or other third party material in this article are included in the article's Creative Commons license, unless indicated otherwise in a credit line to the material. If material is not included in the article's Creative Commons license and your intended use is not permitted by statutory regulation or exceeds the permitted use, you will need to obtain permission directly from the copyright holder. To view a copy of this license, visit http://creativecommons.org/ licenses/by/4.0/

(C) The Author(s) 2021 